\title{
Rough Approximations on Preordered Sets
}

\author{
Yong Chan Kim ${ }^{1}$ and Young Sun $\mathrm{Kim}^{2}$ \\ 1 Department of Mathematics, Gangneung-Wonju National University, Gangneung, 201-702, Korea \\ 2 Department of Applied Mathematics, Pai Chai University, Dae Jeon, 302-735, Korea
}

\begin{abstract}
In this paper, we investigate the properties of rough approximations defined by preordered sets. We study the relations among the lower and upper rough approximations, closure and interior systems, and closure and interior operators.
\end{abstract}

Key Words: Preordered sets, Lower and upper rough approximations, Closure and interior systems, Closure and interior operators.

\section{Introduction and preliminaries}

An information consists of $(X, Y, R)$ where $X$ is a set of objects, $Y$ is a set of attributes and $R$ is a relation between $X$ and $Y$. Rough set theory was introduced by Pawlak [6] to generalize the classical set theory. Rough approximations are defined by a partition of the universe which is corresponding to the equivalence relation about information. J. Järvinen et.al.[3] define rough approximations on preordered relations that are not necessarily equivalence relations. It is an important mathematical tool for data analysis and knowledge processing [1-7].

In this paper, we investigated the properties of rough approximations defined in preordered sets. We study the relations among the lower and upper approximations, closure and interior systems, closure and interior operators. In particular, we investigated the functorial relations among them.

Let $X$ be a set. A relation $e_{X} \subset X \times X$ is called a preorder if it is reflexive and transitive. We can define a preorder $e_{P(X)} \subset P(X) \times P(X)$ as $(A, B) \in e_{P(X)}$ iff $A \subset B$ for $A, B \in P(X)$. If $\left(X, e_{X}\right)$ is a preordered set and we define a function $(x, y) \in e_{X}^{-1}$ iff $(y, x) \in e_{X}$, then $\left(X, e_{X}^{-1}\right)$ is a preordered set.

A function $I: P(X) \rightarrow P(X)$ is called an interior operator on $X$ if it satisfies the following conditions:

(I1) $I(A) \subset A$,

(I2) If $A \subset B$, then $I(A) \subset I(B)$

(I3) $I(I(A)) \supset I(A)$.

The pair $(X, I)$ is called an interior space.

Let $\left(X, I_{X}\right)$ and $\left(Y, I_{Y}\right)$ be interior spaces. A map

Manuscript received Oct. 30, 2011; revised Nov. 12, 2011; accepted Dec. $2,2011$.

This work was supported by the Research Institute of Natural Science of Gangneung-Wonju National University.
$f:\left(X, I_{X}\right) \rightarrow\left(Y, I_{Y}\right)$ is called I-map if $f^{-1}\left(I_{Y}(B)\right) \subset$ $I_{X}\left(f^{-1}(B)\right)$ for each $B \in P(Y)$.

An operator $C: P(X) \rightarrow P(X)$ is called a closure operator on $X$ if it satisfies the following conditions:

(C1) $C(A) \supset A$,

(C2) If $A \subset B$, then $C(A) \subset C(B)$

(C3) $C(C(A)) \subset C(A)$.

The pair $(X, C)$ is called a closure space. Let $\left(X, C_{X}\right)$ and $\left(Y, C_{Y}\right)$ be closure spaces. A map $f:\left(X, C_{X}\right) \rightarrow$ $\left(Y, C_{Y}\right)$ is called C-map if $f\left(C_{X}(A)\right) \subset C_{Y}(f(A))$ for each $A \in P(X)$.

(1) A family $\mathcal{F}=\{A \in P(X)\}$ is called an interior system on $X$ if $\bigcup_{i \in \Gamma} A_{i} \in \mathcal{F}$ for all $A_{i} \in \mathcal{F}$.

(2) A family $\mathcal{G}=\{A \in P(X)\}$ is called a closure system on $X$ if $\bigcap_{i \in \Gamma} A_{i} \in \mathcal{G}$ for all $A_{i} \in \mathcal{G}$.

Definition 1.1. [3-5] Let $\left(X, e_{X}\right)$ be a preodered set. A set $A \in P(X)$ is called $e_{X}$-upper set if $(x \in A \&(x, y) \in$ $\left.e_{X}\right) \rightarrow y \in A$ for $x, y \in X$.

Theorem 1.2. [3-5] Let $\left(X, e_{X}\right)$ be a preordered set. For $A \in P(X)$, we define operations $\left[e_{X}\right],\left\langle e_{X}\right\rangle$ as follows:

$$
\begin{aligned}
& {\left[e_{X}\right](A)=\left\{x \in X \mid(\forall z \in X)\left((x, z) \in e_{X} \rightarrow z \in A\right)\right\}} \\
& \left\langle e_{X}\right\rangle(A)=\left\{x \in X \mid(\exists z \in X)\left((x, z) \in e_{X} \& z \in A\right)\right\} .
\end{aligned}
$$

Then the following properties:

(1)If $\left(e_{X}\right)_{x}=\left\{z \in X \mid(x, z) \in e_{X}\right\}$ and $\left(e_{X}\right)_{x}^{-1}=$ $\left\{z \in X \mid(z, x) \in e_{X}\right\}$, then $\left(e_{X}\right)_{x}$ and $\left(\left(e_{X}\right)_{x}^{-1}\right)^{c}$ are $e_{X}$-upper set.

(2) $A$ is an $e_{X}$-upper set iff $\left[e_{X}\right](A)=A$ iff $\left[e_{X}^{-1}\right]\left(A^{c}\right)=A^{c}$ iff $\left\langle e_{X}^{-1}\right\rangle(A)=A$.

(3) If $A_{i}$ is an $e_{X}$-upper set for all $i \in \Gamma$, then $\bigcup_{i \in \Gamma} A_{i}$ and $\bigcap_{i \in \Gamma} A_{i}$ are $e_{X}$-upper sets.

(4) $\left[e_{X}\right](A)=\bigcup_{i}\left\{A_{i} \mid A_{i} \subset A, A_{i}: e_{X}\right.$ - upper set $\}$.

(5) $\left\langle e_{X}\right\rangle(A)=\bigcap_{i}\left\{A_{i} \mid A \subset A_{i}, A_{i}: e_{X}^{-1}-\right.$ upper set $\}$. 
Definition 1.3. [3] In above theorem, $\left[e_{X}\right](A)$ and $\left\langle e_{X}\right\rangle(A)$ are called rough lower approximation and rough upper approximation, respectively, for $A \in P(X)$ on a preodered set.

If $e_{X}$ is an equivalence relation, $\left[e_{X}\right](A)$ and $\left\langle e_{X}\right\rangle(A)$ are rough lower approximation and rough upper approximation for $A \in P(X)$ in a Pawlak sense [6].

\section{Rough Approximations on Preordered Sets}

Theorem 2.1. Let $\mathcal{F}=\left\{B_{i} \in P(X) \mid i \in \Gamma\right\}$ be an interior system.

$$
\begin{gathered}
I_{\mathcal{F}}(A)=\bigcup\{B \mid B \subset A, B \in \mathcal{F}\} \\
C_{\mathcal{F}^{*}}(A)=\bigcap\left\{B \mid A \subset B, B \in \mathcal{F}^{*}\right\}
\end{gathered}
$$

where $\mathcal{F}^{*}=\left\{A_{i} \in P(X) \mid A_{i}^{c} \in \mathcal{F}\right\}$.

Then; (1) $\mathcal{F}^{*}$ is a closure system.

(2) $I_{\mathcal{F}}$ is an interior operator on $X$ with $\mathcal{F}_{I_{\mathcal{F}}}=\mathcal{F}$ where $\mathcal{F}_{I_{\mathcal{F}}}=\left\{A \in P(X) \mid I_{\mathcal{F}}(A)=A\right\}$.

(3) $C_{\mathcal{F}^{*}}$ is an closure operator on $X$ with $\mathcal{F}_{C_{\mathcal{F}^{*}}}^{*}=\mathcal{F}^{*}$ where $\mathcal{F}_{C_{\mathcal{F}^{*}}}^{*}=\left\{A \in P(X) \mid C_{\mathcal{F}^{*}}(A)=A\right\}$.

(4) There exists a preorder $e_{\mathcal{F}}$ on $X$ such that $\mathcal{F} \subset \mathcal{F}_{\left[e_{\mathcal{F}}\right]}$ with

$$
(x, y) \in e_{\mathcal{F}} \text { iff }\left(\forall B_{i} \in \mathcal{F}\right)\left(x \in B_{i} \rightarrow y \in B_{i}\right) .
$$

In particular, $I_{\mathcal{F}}(A) \subset\left[e_{\mathcal{F}}\right](A)$ with

$$
y \in\left[e_{\mathcal{F}}\right]\left(\{x\}^{c}\right) \text { iff } y \in I_{\mathcal{F}}\left(\{x\}^{c}\right) \text { iff }(y, x) \notin e_{\mathcal{F}} .
$$

(5) There exists a preorder $e_{\mathcal{F}^{*}}$ on $X$ such that $\mathcal{F}^{*} \subset$ $\mathcal{F}_{\left\langle e_{\mathcal{F} *}\right\rangle}^{*}$ with

$$
(x, y) \in e_{\mathcal{F}^{*}} \text { iff }\left(\forall A_{i} \in \mathcal{F}^{*}\right)\left(y \in A_{i} \rightarrow x \in A_{i}\right) .
$$

In particular, $\left\langle e_{\mathcal{F}^{*}}\right\rangle(A) \subset C_{\mathcal{F}^{*}}(A)$ with

$$
y \in\left\langle e_{\mathcal{F}^{*}}\right\rangle(\{x\}) \text { iff } y \in C_{\mathcal{F}^{*}}(\{x\}) \text { iff }(y, x) \in e_{\mathcal{F}^{*}} .
$$

Proof. (1),(2) and (3) are easily proved.

(4) Since $\models\left(x \in B_{j} \rightarrow y \in B_{j}\right) \&\left(y \in B_{j} \rightarrow z \in\right.$ $\left.B_{j}\right) \rightarrow\left(x \in B_{j} \rightarrow z \in B_{j}\right)$, then $e_{\mathcal{F}}$ is transitive. We easily show that $e_{\mathcal{F}}$ is a preorder on $X$. Let $B_{j} \in \mathcal{F}$. Since $\models\left(x \in B_{j}\right) \&\left(x \in B_{j} \rightarrow y \in B_{j}\right) \rightarrow y \in B_{j}$ and $=\left(x \in B_{j}\right) \&(x, y) \in e_{\mathcal{F}} \rightarrow\left(x \in B_{j}\right) \&\left(x \in B_{j} \rightarrow\right.$ $\left.y \in B_{j}\right)$, by M.P., $\models\left(x \in B_{j}\right) \&(x, y) \in e_{\mathcal{F}} \rightarrow y \in B_{j}$. So, $B_{j}$ is $e_{\mathcal{F}}$-upper set. By Theorem $1.2(2), B_{j} \in \mathcal{F}_{\left[e_{\mathcal{F}}\right]}$. Thus, $\mathcal{F} \subset \mathcal{F}_{\left[e_{\mathcal{F}}\right]}$.

Since $I_{\mathcal{F}}(A) \in \mathcal{F}, \models\left(x \in I_{\mathcal{F}}(A)\right) \&\left(x \in I_{\mathcal{F}}(A) \rightarrow\right.$ $y \in I_{\mathcal{F}}(A) \rightarrow y \in I_{\mathcal{F}}(A)$ iff $\mid=\left(x \in I_{\mathcal{F}}(A)\right) \rightarrow((x \in$ $\left.\left.I_{\mathcal{F}}(A) \rightarrow y \in I_{\mathcal{F}}(A)\right) \rightarrow y \in I_{\mathcal{F}}(A)\right)$. Since $\models((x \in$
$\left.\left.\left.I_{\mathcal{F}}(A) \rightarrow y \in I_{\mathcal{F}}(A)\right) \rightarrow y \in I_{\mathcal{F}}(A)\right) \rightarrow x \in\left[e_{\mathcal{F}}\right](A)\right)$, by M.P., $\left.\models x \in I_{\mathcal{F}}(A) \rightarrow x \in\left[e_{\mathcal{F}}\right](A)\right)$.

$$
\begin{aligned}
y \in\left[e_{\mathcal{F}}\right]\left(\{x\}^{c}\right) \quad & \text { iff }(\forall z \in X)\left((y, z) \in e_{\mathcal{F}} \rightarrow z \in\{x\}^{c}\right) \\
y \in I_{\mathcal{F}}\left(\{x\}^{c}\right) \quad & \text { iff }(y, x) \notin e_{\mathcal{F}} . \\
& \text { iff } y \in \bigcup_{B_{i} \in \mathcal{F}} B_{i} \& B_{i} \subset\{x\}^{c} \\
& \text { iff }\left(\exists B_{i} \in \mathcal{F}\right)\left(x \in B_{i}^{c} \& y \in B_{i}\right) \\
& \text { iff } \left.\left(\forall B_{i} \in \mathcal{F}\right)\left(y \in B_{i} \rightarrow x \in B_{i}\right)\right)^{c} \\
& \text { if } \notin e_{\mathcal{F}}
\end{aligned}
$$

(5) We easily show that $e_{\mathcal{F}^{*}}$ is a preorder on $X$. Let $D_{j} \in \mathcal{F}^{*}$. Since $\mid=x \in D_{j} \&(y, x) \in e_{\mathcal{F}^{*}} \rightarrow x \in$ $D_{j} \&\left(x \in D_{j} \rightarrow y \in D_{j}\right)$ and $\models x \in D_{j} \&\left(x \in D_{j} \rightarrow\right.$ $\left.y \in D_{j}\right) \rightarrow y \in D_{j}$, by M.P., $\models x \in D_{j} \&(y, x) \in$ $e_{\mathcal{F}^{*}} \rightarrow y \in D_{j}$. Hence $D_{j}$ is $e_{X}^{-1}$-upper set. By Theorem $1.2(2),\left\langle e_{\left.\mathcal{F}^{*}\right\rangle}\right\rangle\left(D_{j}\right)=D_{j}$. Hence $D_{j} \in \mathcal{F}_{\left\langle e_{\left.\mathcal{F}^{*}\right\rangle}^{*}\right.}^{*}$. Thus, $\mathcal{F}^{*} \subset \mathcal{F}_{\left\langle e_{\mathcal{F} *\rangle}^{*}\right.}^{*}$

Since $C_{\mathcal{F}^{*}}(A) \in \mathcal{F}^{*}$, we have $x \in\left\langle e_{\mathcal{F}^{*}}\right\rangle(A)$ implies $(\exists y \in Y)\left(\left(y \in C_{\mathcal{F}^{*}}(A) \rightarrow x \in C_{\mathcal{F}^{*}}(A)\right) \& y \in A\right)$ implies $(\exists y \in Y)\left(\left(y \in C_{\mathcal{F}^{*}}(A) \rightarrow x \in C_{\mathcal{F}^{*}}(A)\right) \& y \in\right.$ $C_{\mathcal{F}^{*}}(A)$ implies $x \in C_{\mathcal{F}^{*}}(A)$. Thus, $\left\langle e_{\mathcal{F}^{*}}\right\rangle(A) \subset$ $C_{\mathcal{F}^{*}}(A)$.

$$
\begin{aligned}
y \in\left\langle e_{\mathcal{F}^{*}}\right\rangle(\{x\}) \quad & \text { iff }(\exists z \in X)\left((y, z) \in e_{\mathcal{F}^{*}} \& z \in\{x\}\right) \\
& \text { iff }(y, x) \in e_{\mathcal{F}^{*}} . \\
y \in C_{\mathcal{F}^{*}}(\{x\}) \quad & =\bigcap\left\{B \mid\{x\} \subset B, B \in \mathcal{F}^{*}\right\} \\
& \text { iff } x \in B \rightarrow y \in \bigcap_{B \in \mathcal{F}^{*}} B \\
& \text { iff }\left(\forall B \in \mathcal{F}^{*}\right)(x \in B \rightarrow y \in B) \\
& \text { iff }(y, x) \in e_{\mathcal{F}^{*}} .
\end{aligned}
$$

Theorem 2.2. Let $\left(X, e_{X}\right)$ be a preordered set. We define $\mathcal{F}_{\left[e_{X}\right]}, \mathcal{G}_{\left\langle e_{X}\right\rangle}$ as follows:

$$
\begin{aligned}
\mathcal{F}_{\left[e_{X}\right]} & =\left\{A \in P(X) \mid\left[e_{X}\right](A)=A\right\} \\
\mathcal{G}_{\left\langle e_{X}\right\rangle} & =\left\{A \in P(X) \mid\left\langle e_{X}\right\rangle(A)=A\right\}
\end{aligned}
$$

Then; (1) $\left[e_{X}\right]$ is an interior operator on $X$ with $x \in$ $\left[e_{X}\right]\left(\{y\}^{c}\right)$ iff $(x, y) \notin e_{X}$.

(2) $\left\langle e_{X}\right\rangle$ is a closure operator on $X$ with $x \in\left\langle e_{X}\right\rangle(\{y\})$ iff $(x, y) \in e_{X}$.

(3) $\mathcal{F}_{\left[e_{X}\right]}$ is an interior and closure system with $I_{\mathcal{F}_{\left[e_{X}\right]}}=$ $\left[e_{X}\right]$ where $I_{\mathcal{F}_{\left[e_{X}\right]}}(A)=\bigcup\left\{B \mid B \subset A, B \in \mathcal{F}_{\left[e_{X}\right]}\right\}$.

(4) $\mathcal{G}_{\left\langle e_{X}\right\rangle}$ is an interior and closure system with $C_{\mathcal{G}_{\left\langle e_{X}\right\rangle}}=\left\langle e_{X}\right\rangle$ where $C_{\mathcal{G}_{\left\langle e_{X}\right\rangle}}(A)=\bigcap\{B \mid A \subset B, B \in$ $\left.\mathcal{G}_{\left\langle e_{X}\right\rangle}\right\}$.

(5) $e_{\mathcal{F}_{\left[e_{X}\right]}}=e_{X}$ and $e_{\mathcal{G}_{\left\langle e_{X}\right\rangle}}=e_{X}$.

Proof. (1) Since

$$
\begin{aligned}
& x \in\left[e_{X}\right]\left(\left[e_{X}\right](A)\right) \\
& \text { iff }(\forall z \in X)\left((x, z) \in e_{X} \rightarrow z \in\left[e_{X}\right](A)\right) \\
& \text { iff }(\forall z \in X)\left(( x , z ) \in e _ { X } \rightarrow ( \forall w \in X ) \left((z, w) \in e_{X}\right.\right. \\
& \rightarrow w \in A)) \\
& \text { iff }(\forall w \in X)\left((\forall z \in X)\left((x, z) \in e_{X} \&(z, w) \in e_{X}\right)\right. \\
& \rightarrow w \in A)
\end{aligned}
$$


then $(\forall w \in X)\left((x, w) \in e_{X} \rightarrow w \in A\right)$. Hence $\left[e_{X}\right]\left(\left[e_{X}\right](A)\right) \subset\left[e_{X}\right](A)$.

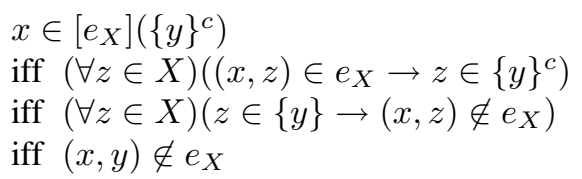

(3) Let $A_{i} \in \mathcal{F}_{\left[e_{X}\right]}$ for $i \in \Gamma$. Since $x \in\left[e_{X}\right]\left(\bigcap_{i \in \Gamma} A_{i}\right)$ iff $(\forall z \in X)\left((x, z) \in e_{X} \rightarrow z \in \bigcap_{i \in \Gamma} A_{i}\right)$ iff $(\forall i \in$ $\Gamma)(\forall z \in X)\left((x, z) \in e_{X} \rightarrow z \in A_{i}\right)$, we have

$$
\left[e_{X}\right]\left(\bigcap_{i \in \Gamma} A_{i}\right)=\bigcap_{i \in \Gamma}\left[e_{X}\right]\left(A_{i}\right)=\bigcap_{i \in \Gamma} A_{i}
$$

Hence $\bigcap_{i \in \Gamma} A_{i} \in \mathcal{F}_{\left[e_{X}\right]}$. Since $\bigcup_{i \in \Gamma} A_{i}=$ $\bigcup_{i \in \Gamma}\left[e_{X}\right]\left(A_{i}\right) \subset\left[e_{X}\right]\left(\bigcup_{i \in \Gamma} A_{i}\right) \subset \bigcup_{i \in \Gamma} A_{i}$, then $\bigcup_{i \in \Gamma} A_{i} \in \mathcal{F}_{\left[e_{X}\right]}$.

Since $\left[e_{X}\right]\left(\left[e_{X}\right](A)\right)=\left[e_{X}\right](A) \subset A, I_{\mathcal{F}_{\left[e_{X}\right]}} \supset\left[e_{X}\right]$.

Since $I_{\mathcal{F}_{\left[e_{X}\right]}}(A) \subset A$ and $I_{\mathcal{F}_{\left[e_{X}\right]}}(A) \in \mathcal{F}_{\left[e_{X}\right]}$, then $\left[e_{X}\right]\left(I_{\mathcal{F}_{\left[e_{X}\right]}}(A)\right)=I_{\mathcal{F}_{\left[e_{X}\right]}}(A) \subset\left[e_{X}\right](A)$.

(4) Let $A_{i} \in \mathcal{G}_{\left\langle e_{X}\right\rangle}$ for $i \in \Gamma$. Since $x \in\left\langle e_{X}\right\rangle\left(\bigcup_{i \in \Gamma} A_{i}\right)$ iff $(\exists z \in X)\left((x, z) \in e_{X} \quad \& z \in \bigcup_{i \in \Gamma} A_{i}\right)$ iff $(\exists i \in$ $\Gamma)(\exists z \in X)\left((x, z) \in e_{X} \& z \in A_{i}\right)$, we have

$$
\left\langle e_{X}\right\rangle\left(\bigcup_{i \in \Gamma} A_{i}\right)=\bigcup_{i \in \Gamma}\left\langle e_{X}\right\rangle\left(A_{i}\right)=\bigcup_{i \in \Gamma} A_{i}
$$

Hence $\bigcup_{i \in \Gamma} A_{i} \in \mathcal{G}_{\left\langle e_{X}\right\rangle}$. Since $\bigcap_{i \in \Gamma}\left(A_{i}\right)=$ $\bigcap_{i \in \Gamma}\left\langle e_{X}\right\rangle\left(A_{i}\right) \supset\left\langle e_{X}\right\rangle\left(\bigcap_{i \in \Gamma} A_{i}\right) \supset \bigcap_{i \in \Gamma} A_{i}$, then $\bigcap_{i \in \Gamma} A_{i} \in \mathcal{G}_{\left\langle e_{X}\right\rangle}$.

Since $\left\langle e_{X}\right\rangle\left(\left\langle e_{X}\right\rangle(A)\right)=\left\langle e_{X}\right\rangle(A) \supset A, C_{\mathcal{G}_{\left\langle e_{X}\right\rangle}} \subset$ $\left\langle e_{X}\right\rangle$.

Since $A \subset C_{\mathcal{G}_{\left\langle e_{X}\right\rangle}}$ and $C_{\mathcal{G}_{\left\langle e_{X}\right\rangle}}(A) \in \mathcal{G}_{\left\langle e_{X}\right\rangle}$, then $C_{\mathcal{G}_{\left\langle e_{X}\right\rangle}}(A)=C_{\mathcal{G}_{\left\langle e_{X}\right\rangle}}(A) \supset\left\langle e_{X}\right\rangle(A)$.

(5) For $B_{i} \in \mathcal{F}_{\left[e_{X}\right]}$, since $\left[e_{X}\right]\left(B_{i}\right)=B_{i}$, by Theorem 1.2(2), $B_{i}$ is $e_{X}$-upper set. Hence $=(x, y) \in$ $e_{X} \rightarrow\left(x \in B_{i} \rightarrow y \in B_{i}\right)$. Since $(x, y) \in e_{\mathcal{F}_{\left[e_{X}\right]}}$ iff $\left(\forall B_{i} \in \mathcal{F}_{\left[e_{X}\right]}\right)\left(x \in B_{i}(x) \rightarrow y \in B_{i}\right)$, then $e_{X} \subset$

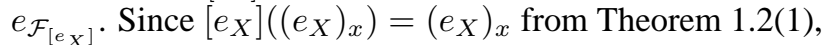
$(x, y) \in e_{\mathcal{F}_{\left[e_{X}\right]}}$ implies $\left(x \in\left(e_{X}\right)_{x} \rightarrow y \in\left(e_{X}\right)_{x}\right)$ implies $(x, y) \in e_{X}$. Hence $e_{\mathcal{F}_{\left[e_{X}\right]}} \subset e_{X}$.

For $B_{i} \in \mathcal{G}_{\left\langle e_{X}\right\rangle}$, since $\left\langle e_{X}\right\rangle\left(B_{i}\right)=B_{i}$, by Theorem 1.2(2), $B_{i}$ is $e_{X}^{-1}$-upper set. Hence $\models(x, y) \in e_{X} \rightarrow$ $\left(y \in B_{i} \rightarrow x \in B_{i}\right)$. Since $(x, y) \in e_{\mathcal{G}_{\left\langle e_{X}\right\rangle}}$ iff $\left(\forall B_{i} \in \mathcal{G}_{\left\langle e_{X}\right\rangle}\right)\left(y \in B_{i} \rightarrow x \in B_{i}\right)$, then $e_{X} \subset e_{\mathcal{G}_{\left\langle e_{X}\right\rangle}}$. Since $\left\langle e_{X}\right\rangle\left(\left(e_{X}\right)_{y}^{-1}\right)=\left(e_{X}\right)_{y}^{-1}$ from Theorem 1.2(1), $(x, y) \in e_{\mathcal{G}_{\left\langle e_{X}\right\rangle}}$ implies $\left(y \in\left(e_{X}\right)_{y}^{-1} \rightarrow x \in\left(e_{X}\right)_{y}^{-1}\right)$ implies $(x, y) \in e_{X}$. Hence $e_{\mathcal{G}_{\left\langle e_{X}\right\rangle}} \subset e_{X}$.

Example 2.3. Let $X=\{a, b, c, d\}$ be a set. We define a preoder $e_{X}$ as follows:

$$
e_{X}=\{(a, a)(a, d),(b, b),(b, d),(c, a),
$$

$$
(c, b),(c, c),(c, d),(d, d)\}
$$

We obtain

$$
\left[e_{X}\right](A)= \begin{cases}\{d\} & \text { if }\{d\} \subset A, \\ \{a, d\} & \text { if }\{a, d\} \subset A,\{b, d\} \not \subset A \\ \{b, d\} & \text { if }\{b, d\} \subset A,\{a, d\} \not \subset A, \\ \{a, b, d\} & \text { if }\{a, b, d\} \subset A, \\ & \{a, d\} \not \subset A,\{b, d\} \not \subset A, \\ X & \text { if } A=X, \\ \emptyset & \text { otherwise, }\end{cases}
$$

$$
\mathcal{F}_{\left[e_{X}\right]}=\{\emptyset,\{d\},\{a, d\},\{b, d\},\{a, b, d\}, X\}
$$

$I_{\mathcal{F}_{\left[e_{X}\right]}}=\left[e_{X}\right]$ and $e_{\mathcal{F}_{\left[e_{X}\right]}}=e_{X}$.

$$
\left\langle e_{X}\right\rangle(A)= \begin{cases}\emptyset & \text { if } A=\emptyset \\ \{c\} & \text { if } A=\{c\}, \\ \{a, c\} & \text { if }\{a\} \subset A, A \not \supset\{b\}, A \not \supset\{d\} \\ \{b, c\} & \text { if }\{b\} \subset A, A \not \supset\{a\}, A \not \supset\{d\} \\ \{a, b, c\} & \text { if }\{a, b\} \subset A, A \not \supset\{d\} \\ X & \text { if }\{d\} \subset A .\end{cases}
$$$$
\mathcal{G}_{\left\langle e_{X}\right\rangle}=\{\emptyset,\{c\},\{a, c\},\{b, c\},\{a, b, c\}, X\}
$$

Moreover, $C_{\mathcal{G}_{\left\langle e_{X}\right\rangle}}=\left\langle e_{X}\right\rangle$ and $e_{\mathcal{G}_{\left\langle e_{X}\right\rangle}}=e_{X}$.

Theorem 2.4. Let $C: P(X) \rightarrow P(X)$ be a closure operator.

Then; (1) $\mathcal{G}_{C}=\{A \in P(X) \mid A=C(A)\}$ is a closure system on $X$ with $C_{\mathcal{G}_{C}}=C$.

(2) Define $(x, y) \in e_{C}$ iff $y \in C(\{x\})$. Then $e_{C}$ is a preorder $e_{C}$ on $X$ with $\left\langle e_{C}\right\rangle(A)=\left\langle e_{\mathcal{G}_{C}}\right\rangle(A) \subset C(A)$ and

$$
y \in\left\langle e_{\mathcal{G}_{C}}\right\rangle(\{x\}) \text { iff } y \in C(\{x\}) \text { iff }(y, x) \in e_{\mathcal{G}_{C}} .
$$

Proof. (1) For each $A_{i} \in \mathcal{G}_{C}, \cap_{i \in \Gamma} A_{i} \in \mathcal{G}_{C}$ because

$\cap_{i \in \Gamma} A_{i} \subset C\left(\cap_{i \in \Gamma} A_{i}\right) \subset \cap_{i \in \Gamma} C\left(A_{i}\right) \subset \cap_{i \in \Gamma} A_{i}$.

Since $C(C(A))=C(A)$, we have $C_{\mathcal{G}_{C}}(A) \subset C(A)$. Since $A \subset C_{\mathcal{G}_{C}}(A), C(A)=C_{\mathcal{G}_{C}}(C(A)) \subset$ $C_{\mathcal{G}_{C}}\left(C_{\mathcal{G}_{C}}(A)\right)=C_{\mathcal{G}_{C}}(A)$.

(2) Since $x \in C(\{x\})$, then $(x, x) \in e_{C}$. For $(x, y) \in$ $e_{C}$ and $(y, z) \in e_{C}$, since $x \in C(\{y\})$ and $y \in C(\{z\})$, then $C(\{y\}) \subset C(C(\{z\}))=C(\{z\})$. Hence $x \in$ $C(\{z\}) ;$ i.e. $(x, z) \in e_{C}$.

Since $C_{\mathcal{G}_{C}}=C$ from (1), $C(\{x\})=\bigcap\{A \mid\{x\} \subset$ $A, A \in \mathcal{G}_{C}$. Then $y \in C(\{x\})$ iff $\models\left(\forall A \in \mathcal{G}_{C}\right)(x \in$ $A \rightarrow y \in A)$ iff $(y, x) \in e_{\mathcal{G}_{C}}$ iff $y \in\left\langle e_{\mathcal{G}_{C}}\right\rangle(\{x\})$ from Theorem 2.2(2). By a similar method in Theorem 2.1(5), $\left\langle e_{\mathcal{G}_{C}}\right\rangle(A) \subset C(A)$.

Theorem 2.5. Let $I: P(X) \rightarrow P(X)$ be an interior operator.

Then; (1) $\mathcal{F}_{I}=\{A \in P(X) \mid A=I(A)\}$ is an interior system on $X$ with $I_{\mathcal{F}_{I}}=I$.

(2) Define $(x, y) \in e_{I}$ iff $x \in\left(I\left(\{x\}^{c}\right)\right)^{c}$. Then $e_{I}$ is a preorder $e_{I}$ on $X$ with $I(A) \subset\left[e_{I}\right](A)=\left[e_{\mathcal{F}_{I}}\right](A)$ and

$y \notin\left[e_{\mathcal{F}_{I}}\right\rangle\left(\{x\}^{c}\right)$ iff $y \notin I\left(\{x\}^{c}\right)$ iff $(y, x) \notin e_{\mathcal{F}_{I}}$. 
Proof. (1) It is similarly proved as in Theorem 2.4(1).

(2) Since $x \in\left(I\left(\{x\}^{c}\right)\right)^{c}$ iff $\{x\} \subset\left(I\left(\{x\}^{c}\right)\right)^{c}$ iff $I\left(\{x\}^{c}\right) \subset\{x\}^{c}$, then $(x, x) \in e_{I}$. For $(x, y) \in e_{I}$ and $(y, z) \in e_{I}$, since $I\left(\{y\}^{c}\right) \subset\{x\}^{c}$ and $I\left(\{z\}^{c}\right) \subset\{y\}^{c}$, then $I\left(\{z\}^{c}\right) \subset I\left(I\left(\{z\}^{c}\right)\right) \subset I\left(\{y\}^{c}\right) \subset\{x\}^{c}$. Hence $x \in\left(I\left(\{z\}^{c}\right)\right)^{c}$;i.e. $(x, z) \in e_{I}$.

Since $I_{\mathcal{F}_{I}}=I$ from (1), $I\left(\{x\}^{c}\right)^{c}=\bigcap\left\{A^{c} \mid\{x\} \subset\right.$ $\left.A^{c}, A \in \mathcal{F}_{I}\right\}$. Then $y \in\left(I\left(\{x\}^{c}\right)\right)^{c}$ iff $\models\left(\forall A \in \mathcal{F}_{I}\right)(x \in$ $\left.A^{c} \rightarrow y \in A^{c}\right)$ iff $\models\left(\forall A \in \mathcal{F}_{I}\right)(y \in A \rightarrow x \in A)$ iff $(y, x) \in e_{\mathcal{F}_{I}}$. Moreover, $y \in\left[e_{\mathcal{F}_{I}}\right]\left(\{x\}^{c}\right)$ iff $=(\forall z \in$ $X)\left((y, z) \in e_{\mathcal{F}_{I}} \rightarrow z \in\{x\}^{c}\right)$ iff $(y, x) \notin e_{\mathcal{F}_{I}}$. Вy a similar method in Theorem 2.1(4), $I(A) \subset\left[e_{\mathcal{F}_{I}}\right]$.

Example 2.6. Let $X=\{a, b, c, d\}, B_{1}=\{a, b\}$ and $B_{2}=$ $\{b, c\}$ be sets. We define functions $I, C: P(X) \rightarrow P(X)$ as follows:

$$
\begin{aligned}
& I(A)= \begin{cases}B_{1} & \text { if } B_{1} \subset A, \\
B_{2} & \text { if } B_{2} \subset A, \\
B_{1} \cup B_{2} & \text { if } B_{1} \cup B_{2} \subset A, \\
\emptyset & B_{1} \not \subset A, B_{2} \not \subset A, \\
\text { otherwise, }\end{cases} \\
& C(A)= \begin{cases}B_{1} & \text { if } A \subset B_{1}, A \not \subset B_{2} \\
B_{2} & \text { if } A \subset B_{2}, A \not \subset B_{2} \\
B_{1} \cap B_{2} & \text { if } A \subset B_{1} \cap B_{2}, \\
X & \text { otherwise. }\end{cases}
\end{aligned}
$$

(1) $I$ is an interior operator on $X$ with $\mathcal{F}_{I}=\{A \mid$ $I(A)=A\}=\left\{\emptyset, B_{1}, B_{2}, B_{1} \cup B_{2}\right\}$.

(2) $C$ is a closure operator on $X$ with $\mathcal{G}_{C}=\{A \mid$ $C(A)=A\}=\left\{B_{1}, B_{2}, B_{1} \cap B_{2}, X\right\}$.

(3) $I_{\mathcal{F}_{I}}=I$ and $C_{\mathcal{G}_{C}}=C$.

(4) There exists a preorder $e_{\mathcal{F}_{I}}$ on $X$ such that $\mathcal{F}_{I} \subset$ $\mathcal{F}_{\left[e_{\mathcal{F}_{I}}\right]}=\left\{\emptyset, B_{1}, B_{2}, B_{1} \cap B_{2}, B_{1} \cup B_{2}, X\right\}$ with

$$
\begin{gathered}
(x, y) \in e_{\mathcal{F}_{I}} \text { iff } \models\left(\forall B \in \mathcal{F}_{I}\right)(x \in B \rightarrow y \in B) \\
e_{\mathcal{F}_{I}}=\{(a, a)(a, b),(b, b),(c, b),(c, c), \\
(d, a),(d, b),(d, c),(d, d)\}
\end{gathered}
$$$$
\left[e_{\mathcal{F}_{I}}\right](A)= \begin{cases}B_{1} \cap B_{2} & \text { if } B_{1} \cap B_{2} \subset A, \\ B_{1} & \text { if } B_{1} \subset A, B_{2} \not \subset A \\ B_{2} & \text { if } B_{2} \subset A, B_{1} \not \subset A, \\ B_{1} \cup B_{2} & \text { if } B_{1} \cup B_{2} \subset A, \\ & B_{1} \not \subset A, B_{2} \not \subset A, \\ X & \text { if } A=X, \\ \emptyset & \text { otherwise, }\end{cases}
$$

Hence $I(A) \subset\left[e_{\mathcal{F}_{I}}\right](A)$ with $y \in\left[e_{\mathcal{F}_{I}}\right]\left(\{x\}^{c}\right)$ iff $y \in$ $I\left(\{x\}^{c}\right)$ iff $(y, x) \notin e_{\mathcal{F}_{I}}=e_{I}$ because

$$
\begin{aligned}
& {\left[e_{\mathcal{F}_{I}}\right]\left(\{a\}^{c}\right)=I\left(\{a\}^{c}\right)=\{b, c\}} \\
& {\left[e_{\mathcal{F}_{I}}\right]\left(\{b\}^{c}\right)=I\left(\{b\}^{c}\right)=\emptyset} \\
& {\left[e_{\mathcal{F}_{I}}\right]\left(\{c\}^{c}\right)=I\left(\{c\}^{c}\right)=\{a, b\}} \\
& {\left[e_{\mathcal{F}_{I}}\right]\left(\{d\}^{c}\right)=I\left(\{d\}^{c}\right)=\{a, b, c\}}
\end{aligned}
$$

(5) There exists a preorder $e_{\mathcal{G}_{C}}$ on $X$ such that $\mathcal{G}_{C} \subset$ $\mathcal{G}_{\left\langle e_{\mathcal{G}_{C}}\right\rangle}=\left\{\emptyset, B_{1}, B_{2}, B_{1} \cap B_{2}, B_{1} \cup B_{2}, X\right\}$ with

$$
\begin{gathered}
(x, y) \in e_{\mathcal{G}_{C}} \text { iff } \models\left(\forall B_{i} \in \mathcal{G}_{C}\right)\left(y \in B_{i} \rightarrow x \in B_{i}\right) \\
e_{\mathcal{G}_{C}}=\{(a, a)(a, d),(b, a),(b, b),(b, c),(b, d) \\
(c, c),(c, d),(d, d)\} \\
\left\langle e_{\mathcal{G}_{C}}\right\rangle(A)= \begin{cases}\emptyset & \text { if } A=\emptyset \\
B_{1} & \text { if } A \subset B_{1}, A \not \subset B_{2} \\
B_{2} & \text { if } A \subset B_{2}, A \not \subset B_{2} \\
B_{1} \cap B_{2} & \text { if } \emptyset \neq A \subset B_{1} \cap B_{2}, \\
B_{1} \cup B_{2} & \text { if } A \subset B_{1} \cup B_{2}, \\
X & A \not \subset B_{1}, A \not \subset B_{2}\end{cases}
\end{gathered}
$$

Hence $\left\langle e_{\mathcal{G}_{C}}\right\rangle(A) \subset C(A)$ with $y \in\left\langle e_{\mathcal{G}_{C}}\right\rangle(\{x\})$ iff $y \in$ $C(\{x\})$ iff $(y, x) \in e_{\mathcal{G}_{C}}=e_{C}$.

$$
\begin{aligned}
& \left\langle e_{\mathcal{G}_{C}}\right\rangle(\{a\})=C(\{a\})=\{a, b\} \\
& \left\langle e_{\mathcal{G}_{C}}\right\rangle(\{b\})=C(\{b\})=\{b\} \\
& \left\langle e_{\mathcal{G}_{C}}\right\rangle(\{c\})=C(\{c\})=\{b, c\} \\
& \left\langle e_{\mathcal{G}_{C}}\right\rangle(\{d\})=C(\{d\})=\{a, b, c, d\}
\end{aligned}
$$

Theorem 2.7. Let $\left(X, e_{X}\right)$ and $\left(Y, e_{Y}\right)$ be preordered sets. Then the following statements are equivalent:

(1) $f:\left(X, e_{X}\right) \rightarrow\left(Y, e_{Y}\right)$ is an order preserving map,

(2) $f^{-1}(B)$ is an $e_{X}$-upper set for $e_{Y}$-upper set $B$,

(3) $f^{-1}(D)$ is an $e_{X}^{-1}$-upper set for $e_{Y}^{-1}$-upper set $D$,

(4) $f\left(\left\langle e_{X}\right\rangle(A)\right) \subset\left\langle e_{Y}\right\rangle(f(A))$, for each $A \in P(X)$,

(5) $f\left(\left[e_{X}\right](A)\right) \subset\left[e_{Y}\right](f(A))$, for each $A \in P(X)$,

(6) $f^{-1}\left(\left[e_{Y}\right](B)\right) \subset\left[e_{X}\right]\left(f^{-1}(B)\right)$, for each $B \in$ $P(Y)$.

(7) $f^{-1}\left(\left\langle e_{Y}\right\rangle(B)\right) \subset\left\langle e_{X}\right\rangle\left(f^{-1}(B)\right)$, for each $B \in$ $P(Y)$.

Proof. $(1) \Rightarrow(2)$. Let $B$ be an $e_{Y}$-upper set. Since $f$ is an order preserving map, $\models x \in f^{-1}(B) \&(x, z) \in e_{X} \rightarrow$ $f(x) \in B \&(f(x), f(z)) \in e_{Y}$ and $\models(f(x), f(z)) \in$ $e_{Y} \& f(z) \in B \rightarrow z \in f^{-1}(B)$. By M.P., $\models x \in$ $f^{-1}(B) \&(x, z) \in e_{X} \rightarrow z \in f^{-1}(B)$. Hence $f^{-1}(B)$ is an $e_{X}$-upper set.

$(2) \Rightarrow(4)$. Since $B_{i}$ is an $e_{Y}^{-1}$-upper set iff $B_{i}^{c}$ is an $e_{Y^{-}}$ upper set, then $f^{-1}\left(B_{i}^{c}\right)=\left(f^{-1}\left(B_{i}\right)\right)^{c}$ is an $e_{X}$-upper set iff $f^{-1}\left(B_{i}\right)$ is an $e_{X}^{-1}$-upper set. Thus,

$$
\begin{aligned}
&\left\langle e_{Y}\right\rangle(f(A)) \\
&= \bigcap_{i}\left\{B_{i} \mid f(A) \subset B_{i}, B_{i}: e_{Y}^{-1}-\text { upper set }\right\} \\
&= \bigcap_{i}\left\{B_{i} \mid A \subset f^{-1}\left(B_{i}\right), B_{i}: e_{Y}^{-1}-\text { upper set }\right\} \\
& \supset \bigcap_{i}\left\{f\left(f^{-1}\left(B_{i}\right)\right) \mid A \subset f^{-1}\left(B_{i}\right),\right. \\
&\left.f^{-1}\left(B_{i}\right): e_{X}^{-1}-\text { upper set }\right\} \\
& \supset f\left(\bigcap _ { i } \left\{f^{-1}\left(B_{i}\right) \mid A \subset f^{-1}\left(B_{i}\right),\right.\right. \\
&\left.f^{-1}\left(B_{i}\right): e_{X}^{-1}-\text { upper set }\right\} \\
& \supset f\left(\left\langle e_{X}\right\rangle(A)\right)
\end{aligned}
$$


(4) $\Rightarrow$ (6). Put $A=f^{-1}(B) . \quad f\left(\left\langle e_{X}\right\rangle\left(f^{-1}(B)\right) \subset\right.$ $\left\langle e_{Y}\right\rangle\left(f\left(f^{-1}(B)\right)\right) \subset\left\langle e_{Y}\right\rangle(B)$ implies $\left\langle e_{X}\right\rangle\left(f^{-1}(B)\right) \subset$ $f^{-1}\left(\left\langle e_{Y}\right\rangle(B)\right) . \quad\left(\left[e_{X}\right]\left(f^{-1}\left(B^{c}\right)\right)\right)^{c} \supset\left(f^{-1}\left(\left[e_{Y}\right]\left(B^{c}\right)\right)\right)^{c}$ implies $\left[e_{X}\right]\left(f^{-1}\left(B^{c}\right)\right) \supset f^{-1}\left(\left[e_{Y}\right]\left(B^{c}\right)\right)$.

(6) $\Rightarrow(1)$. Put $B=\{f(z)\}^{c}$. Since

$f^{-1}\left(\left[e_{Y}\right]\left(\{f(z)\}^{c}\right) \subset\left[e_{X}\right]\left(f^{-1}\left(\left(\{f(z)\}^{c}\right)\right) \subset\left[e_{X}\right]\left(\left(\{z\}^{c}\right)\right.\right.\right.$

Then $(f(x), f(z)) \notin e_{Y}$ iff $x \in f^{-1}\left(\left[e_{Y}\right]\left(\{f(z)\}^{c}\right)\right)$ implies $x \in\left[e_{X}\right]\left(f^{-1}\left(\{f(z)\}^{c}\right)\right)$ implies $x \in\left[e_{X}\right]\left(\{z\}^{c}\right)$ iff $(x, z) \notin e_{X}$.

Similarly, (1) $\Rightarrow(3) \Rightarrow(5) \Rightarrow(7) \Rightarrow(1)$.

Theorem 2.8. Let $\left(X, \mathcal{G}_{X}\right)$ and $\left(Y, \mathcal{G}_{Y}\right)$ be closure systems. Then the following statements are equivalent:

(1) $f^{-1}(B) \in \mathcal{G}_{X}$ for each $B \in \mathcal{G}_{Y}$,

(2) $f:\left(X, C_{\mathcal{G}_{X}}\right) \rightarrow\left(X, C_{\mathcal{G}_{Y}}\right)$ is a C-map.

(3) $C_{\mathcal{G}_{X}}\left(f^{-1}(B)\right) \subset f^{-1}\left(C_{\mathcal{G}_{Y}}(B)\right)$ for each $B \in$ $P(Y)$.

(4) $f:\left(X, I_{\mathcal{G}_{X}^{*}}\right) \rightarrow\left(X, I_{\mathcal{G}_{Y}^{*}}\right)$ is an I-map.

Moreover, if $f^{-1}(B) \in \mathcal{G}_{X}$ for each $B \in \mathcal{G}_{Y}$, then $f$ : $\left(X, e_{\mathcal{G}_{X}}\right) \rightarrow\left(Y, e_{\mathcal{G}_{Y}}\right)$ is an order preserving map.

Proof. We easily proved that the statements (1), (2),(3) and (4) are equivalent. Let $f^{-1}(B) \in \mathcal{G}_{X}$ for each $B \in \mathcal{G}_{Y}$. Then

$$
\begin{aligned}
& (f(x), f(y)) \in e_{\mathcal{G}_{Y}} \\
& \text { iff }\left(\forall B \in \mathcal{G}_{Y}\right)(f(y) \in B \rightarrow f(x) \in B) \\
& \text { iff }\left(\forall B \in \mathcal{G}_{Y}\right)\left(y \in f^{-1}(B) \rightarrow x \in f^{-1}(B)\right)
\end{aligned}
$$

Hence

$$
\begin{array}{ll}
(x, y) \in \mathcal{G}_{X} \quad \text { iff }\left(\forall D \in \mathcal{G}_{Y}\right)(y \in D \rightarrow x \in D) \\
& \text { implies }(f(x), f(y)) \in e_{\mathcal{G}_{Y}} .
\end{array}
$$

Theorem 2.9. Let $\left(X, C_{X}\right)$ and $\left(Y, C_{Y}\right)$ be closure spaces. Then the following statements are equivalent:

(1) $f: X \rightarrow Y$ is a C-map.

(2) $C_{X}\left(f^{-1}(B)\right) \subset f^{-1}\left(C_{Y}(B)\right)$ for each $B \in L^{Y}$.

(3) $f^{-1}(B) \in \mathcal{G}_{C_{X}}$ for each $B \in \mathcal{G}_{C_{Y}}$,

Moreover, if $f: X \rightarrow Y$ is a C-map, then $f$ : $\left(X, e_{C_{X}}\right) \rightarrow\left(Y, e_{C_{Y}}\right)$ is an order preserving map. If $C_{X}\left(\cup_{i \in \Gamma} A_{i}\right)=\cup_{i \in \Gamma} C_{X}\left(A_{i}\right)$, the converse holds.

Proof. We easily proved that the statements (1), (2) and (3) are equivalent. Since $f\left(C_{X}(\{x\})\right) \subset C_{Y}(\{f(x)\})$, then $z \in C_{X}(\{x\})$ implies $f(z) \in C_{Y}(\{f(x)\})$. Hence $(z, x) \in e_{C_{X}}$ implies $(f(z), f(x)) \in e_{C_{Y}}$.

Since $(z, x) \in e_{C_{X}}$ iff $z \in C_{X}(\{x\})$ implies $(f(z), f(x))$ iff $f(z) \in C_{Y}(\{f(x)\})$, then $f\left(C_{X}(\{x\}) \subset\right.$ $C_{Y}(\{f(x)\})$. For each $A=\cup_{x \in A}\{x\}$,

$$
\begin{gathered}
f(C(A))=f\left(C_{X}\left(\cup_{x \in A}\{x\}\right)=\cup_{x \in A} f\left(C_{X}(\{x\})\right.\right. \\
\subset \cup_{x \in A} C_{Y}(\{f(x)\}) \subset C_{Y}\left(\cup_{x \in A}\{f(x)\}\right)=C_{Y}(f(A))
\end{gathered}
$$

Theorem 2.10. Let $\left(X, I_{X}\right)$ and $\left(Y, I_{Y}\right)$ be interior spaces. Then the following statements are equivalent:

(1) $f: X \rightarrow Y$ is an I-map.

(2) $f^{-1}(B) \in \mathcal{F}_{I_{X}}$ for each $B \in \mathcal{F}_{I_{Y}}$,

Moreover, if $f: X \rightarrow Y$ is an I-map, then $f:$ $\left(X, e_{I_{X}}\right) \rightarrow\left(Y, e_{I_{Y}}\right)$ is an order preserving map. If $I_{X}\left(\cap_{i \in \Gamma} A_{i}\right)=\cap_{i \in \Gamma} I_{X}\left(A_{i}\right)$, the converse holds.

Proof. We easily proved that the statements (1) and (2) are equivalent. Let $f: X \rightarrow Y$ be an I-map. Then

$$
f^{-1}\left(I_{Y}\left(\{f(x)\}^{c}\right)\right) \subset I_{X}\left(f^{-1}\left(\{f(x)\}^{c}\right)\right) \subset I_{X}\left(\{x\}^{c}\right) .
$$

Hence $z \in I_{X}\left(\{x\}^{c}\right)^{c}$ implies $f(z) \in I_{Y}\left(\{f(x)\}^{c}\right)^{c}$. Thus, $(z, x) \in e_{I_{X}}$ implies $(f(z), f(x)) \in e_{I_{Y}}$.

Since $(x, z) \in e_{I_{X}}$ iff $x \in I_{X}\left(\{z\}^{c}\right)^{c}$ implies $(f(x), f(z))$ iff $f(x) \in I_{Y}\left(\left\{\{f(z)\}^{c}\right)^{c}\right.$, then $f\left(I_{X}\left(\{x\}^{c}\right)^{c}\right) \subset I_{Y}\left(\{f(x)\}^{c}\right)^{c}$. Thus $I_{X}\left(\{x\}^{c}\right)^{c} \subset f^{-1}\left(I_{Y}\left(\{f(x)\}^{c}\right)^{c}\right) \quad$ implies $f^{-1}\left(I_{Y}\left(\{f(x)\}^{c}\right)\right) \subset I_{X}\left(\{x\}^{c}\right)$. Put $f(x)=y$. Then $f^{-1}\left(I_{Y}\left(\{y\}^{c}\right)\right) \subset I_{X}\left(\left\{f^{-1}(y)\right\}^{c}\right)$. For each $B=\cap_{y \in B^{c}}\{y\}^{c}$,

$$
\begin{aligned}
f^{-1}\left(I_{Y}(B)\right) & =f^{-1}\left(I_{Y}\left(\cap_{y \in B^{c}}\{y\}^{c}\right)\right) \\
& \subset \cap_{y \in B^{c}} f^{-1}\left(I_{Y}\left(\{y\}^{c}\right)\right) \\
& \subset \cap_{y \in B^{c}} I_{X}\left(f^{-1}\left(\{y\}^{c}\right)\right) \\
& =I_{X}\left(\cap_{y \in B^{c}} f^{-1}\left(\{y\}^{c}\right)\right) \\
& =I_{X}\left(f^{-1}\left(\cap_{y \in B^{c}}\{y\}^{c}\right)\right)=I_{X}\left(f^{-1}(B)\right) .
\end{aligned}
$$

\section{References}

[1] R. Bělohlávek, "Lattices of fixed points of Galois connections," Math. Logic Quart., vol.47, pp.111-116, 2001.

[2] G. Georgescu, A. Popescue, "Non-dual fuzzy connections," Arch. Math. Log., vol. 43, pp.1009-1039, 2004.

[3] J. Järvinen, M. Kondo, J. Kortelainen, ’Logics from Galois connections," Int. J. Approx. Reasoning, vol.49, pp.595-606, 2008.

[4] Y.C. Kim, Y.S. Kim, "Relations and upper sets on partially ordered sets," Int. Math. Forum, vol. 6 , no.19, pp.899-908, 2011.

[5] Y.C. Kim, Y.S. Kim, "Various connections on interior sets," Int. Math. Forum, vol.6, no.19, pp. 909$920,2011$.

[6] Z. Pawlak," Rough sets," Int. J. Comput. Inf. Sci., vol.11, pp.341-356, 1982.

[7] G. Qi and W. Liu," Rough operations on Boolean algebras," Information Sciences, vol. 173, pp.49-63, 2005. 
International Journal of Fuzzy Logic and Intelligent Systems, vol.11 , no. 4, December 2011

[8] R. Wille, Restructuring lattice theory; an approach based on hierarchies of concept, in: 1. Rival(Ed.), Ordered Sets, Reidel, Dordrecht, Boston, 1982.

Yong Chan Kim

Professor of Gangneung-Wonju University

Research Area: Fuzzy topology, Fuzzy logic.

\section{E-mail: yck@gwnu.ac.kr}

\section{Young Sun Kim}

Professor of Pai Chai University

Research Area: Fuzzy topology, Fuzzy logic.

E-mail: yskim@pcu.ac.kr 